\title{
O uso da instrumentação tecnológica em projetos de tecnologia assistiva: captura de movimentos e termografia infravermelha
}

\section{The use of technological instrumentation in assistive technology projects: motion capture and infrared termography}

Eugenio Andrés Díaz Merino ${ }^{1}$

Franciele Forcelini ${ }^{2}$

Thiago Varnier ${ }^{3}$

Giselle Schmidt Alves Díaz Merino ${ }^{4}$ 


\section{Resumo}

O uso de instrumentos tecnológicos em pesquisas tem-se mostrado um recurso auxiliar na obtenção de dados. Portanto, este artigo tem como objetivo identificar protocolos, vantagens e desvantagens a respeito da utilização da captura de movimentos (Xsens) e a termografia infravermelha em estudos de casos em tecnologia assistiva e em pesquisas científicas gerais. Para tanto, foi utilizada a pesquisa aplicada, qualitativa e de cunho descritivo. Os resultados apontam que a instrumentação tecnológica, seguindo protocolos rigorosos, permite a obtenção de dados quantificáveis e confiáveis, mantendo o rigor científico. Logo, se mostra adequada em coletas envolvendo pessoas com limitações físicas e psíquicas, respeitando esta condição mesmo quando a comunicação com os sujeitos encontra-se comprometida. Contudo, entende-se que a captura de movimentos (Xsens) e a termografia infravermelha são métodos viáveis para o desenvolvimento de soluções eficientes, confortáveis e seguras de tecnologias assistivas.

Palavras-chave: Instrumentação tecnológica; tecnologia assistiva; captura de movimentos; termografia infravermelha

\section{Abstract}

The use of technological tools in research has shown to be an auxiliary resource in obtaining data. Therefore, this article aims to identify protocols, advantages and disadvantages regarding the use of motion capture (Xsens) and infrared thermography in case studies in assistive technology and general scientific research. For that, the applied, qualitative and descriptive research was used. The results indicate that the technological instrumentation, following strict protocols, allows the obtaining of quantifiable and reliable data, maintaining the scientific rigor. Therefore, it is adequate in collections involving people with physical and psychic limitations, respecting this condition even when communication with the subjects is compromised. However, it is understood that motion capture (Xsens) and infrared thermography are viable methods for the development of efficient, comfortable and safe solutions of assistive technologies.

Key-words: Technological instrumentation; assistive technology; motion capture; infrared thermography

ISSN: 2316-7963

\footnotetext{
Doutor em Engenharia, UFSC (eugenio.merino@ufsc.br)

${ }^{2}$ Mestranda em Design, UFSC (francieleforcelini@gmail.com)

${ }^{3}$ Mestrando em Design, UFSC (thiagovarnier1@gmail.com)

${ }^{4}$ Doutora em Engenharia, UFSC (gisellemerino@gmail.com)
} 


\section{INTRODUÇÃO}

Em âmbito mundial, 1 (um) em cada 7 (sete) indivíduos possuem algum tipo de deficiência (sensorial, motora e/ou cognitiva) que, no Brasil, corresponde a 45 milhões de indivíduos (BRASIL, 2012; WHO, 2012). Diante disso, nota-se a necessidade do uso e do desenvolvimento de tecnologias assistivas (TA), as quais são entendidas como produtos, recursos, estratégias, práticas, processos, métodos e serviços com a finalidade de promover a funcionalidade de indivíduos com deficiência ou mobilidade reduzida, visando sua autonomia, independência, qualidade de vida e inclusão social (BRASIL, 2015).

Com este propósito, projetos são desenvolvidos para auxiliar indivíduos com diferentes incapacidades, porém os profissionais envolvidos podem se deparar com dificuldades na obtenção de dados precisos e objetivos, devido às próprias limitações (físicas e psíquicas) dos sujeitos dos estudos (MERINO et al., 2017; SPECK et al., 2016).

Neste contexto, a incorporação de instrumentos tecnológicos na coleta de dados pode auxiliar na aferição de dados quantificáveis e confiáveis, principalmente em casos onde a falta de comunicação com os sujeitos impede o acesso às informações (MERINO et al., 2017). Ainda, os autores ressaltam que o objetivo da instrumentação consiste na obtenção de medições quantitativas (físicas, biomecânicas e fisiológicas) dos sujeitos, a fim de caracterizar suas necessidades e disfunções. Desta forma, torna-se um recurso em projetos de TA, gerando dados e informações precisas que podem auxiliar no desenvolvimento de soluções mais eficientes, com condições de conforto e usabilidade.

Dentre os instrumentos existentes, este artigo destaca a aplicação da captura de movimentos e da termografia infravermelha em projetos. A captura de movimentos possibilita a simulação de movimentos humanos, e foi introduzida na indústria como uma ferramenta para facilitar processos de projetos, acelerando seu desenvolvimento e eficiência em custos (LÄMKULL et al., 2009). Já a termografia infravermelha registra as qualidades térmicas superficiais dos objetos por meio da captação da radiação infravermelha e conversão em uma imagem térmica, que exibe as variações de temperatura por um espectro de cores, imperceptíveis a olho nu (MOBLEY, 2002).

Diante do exposto e considerando a importância da instrumentação tecnológica, este artigo propõe-se responder à questão de pesquisa - como a instrumentação tecnológica pode ser utilizada no levantamento de dados para o desenvolvimento de projetos de TA? Portanto, tem como objetivo identificar protocolos, vantagens e desvantagens a respeito da utilização da captura de movimentos (Xsens) e a termografia infravermelha em estudos de casos em TA desenvolvidos pelo NGD/LDU ${ }^{1}$, os quais fazem parte da Rede de Pesquisa e Desenvolvimento em Tecnologia Assistiva (RPDTA) ${ }^{2}$, que visa fomentar e consolidar a área de

1 NGD/LDU - Alocado no Centro de Comunicação e Expressão (CCE) da Universidade Federal de Santa Catarina (UFSC). Conta com uma equipe de pesquisadores e acadêmicos de todos os níveis. Além disso, possui suporte tecnológico do LDU, laboratório integrado que oferece instrumentos tecnológicos como a captura de movimentos, a termografia infravermelha, o rastreamento ocular, a eletromiografia de superfície, a dinamometria digital, a goniometria, dentre outros.

2 RPDTA - Rede de cooperação entre 5 universidades brasileiras: Universidade Federal do Paraná (UFPR), Universidade Federal de Santa Catarina (UFSC), Universidade do Estado de Santa Catarina (UDESC), Universidade Tecnológica Federal do Paraná (UTFPR) e a Universidade Estadual Paulista - Campus Bauru (UNESP-Bauru), e seus respectivos Programas de Pós-Graduação em Engenharia Mecânica, Design e Engenharia de Produção. 
Pesquisa em TA, e também projetos gerais de pesquisa cientificas. Para tanto, foi utilizada a pesquisa de natureza aplicada, com abordagem qualitativa, de cunho descritivo.

Esta pesquisa justifica-se pela exigência por resultados precisos e objetivos em projetos profissionais e de pesquisa, cujo processo de coleta de dados pode ser auxiliado pelo uso de instrumentos tecnológicos. Estes recursos permitem a obtenção de dados quantitativos, com maior confiabilidade e menor subjetividade.

Neste sentido, Merino et al. (2017) destacam, a partir das suas experiências, a importância do uso de equipamentos na obtenção de dados objetivos sobre os usuários, principalmente em casos de TA, onde a comunicação é comprometida (por exemplo: fala, preenchimento de questionários, entrevistas, entre outros), assim o levantamento de informações fica comprometido e até inviabilizado dependendo do tipo de limitação do sujeito. Speck et al. (2016) também aborda a importância dos dados quantitativos na concepção e avaliação de novos produtos mais adequados e satisfatórios aos usuários, reduzindo assim a necessidade de reprojetos.

Logo, a utilização da captura de movimentos e da termografia infravermelha podem apresentar informações e definições precisas sobre os contextos de estudo, auxiliando nas análises dos dados. Estes instrumentos permitem um mapeamento rápido, seguro e preciso e destacam-se pela sua portabilidade, que permite flexibilidade nas aferições (FLIR, 2016b; XSENS, 2012). A captura de movimentos oferece dados dos movimentos de segmentos e articulações corporais (XSENS, 2012) enquanto a termografia infravermelha registra as qualidades térmicas superficiais dos objetos (MOBLEY, 2002), ampliando a visibilidade dos fenômenos, visto que permite uma exploração aprofundada dos objetos de estudo (SILVA; TARALLI; MELZ, 2015).

Todavia, o interesse pela compreensão e a quantificação dos movimentos e temperaturas é demonstrado em diferentes áreas de conhecimento, como a medicina, veterinária, engenharia, design, ergonomia, entre outras (FLIR, 2016a; XSENS, 2012). Além disso, a instrumentação tecnológica vem sendo difundida no meio acadêmico por meio de projetos de pesquisa (GARCÍA et al., 2016; MERINO et al, 2017; SPECK et al., 2016).

\section{FUNDAMENTAÇÃO TEÓRICA}

Neste tópico são apresentados os resultados da pesquisa bibliográfica referente a instrumentação tecnológica - captura de movimentos e termografia infravermelha -, abordando seus conceitos, históricos e características.

\subsection{Captura de movimentos}

O sistema de Captura de Movimentos, também conhecido como Motion Tracking ou pela abreviação MoCap trata-se de uma técnica utilizada para mapear os movimentos realizados por um objeto e reproduzi-los em ambiente digital (GOMIDE et al., 2009). É entendido como um conceito utilizado em biomecânica para descrever os sistemas com a finalidade de registrar o movimento de um corpo por meio de algum dispositivo e a partir destes dados registrados, calcular as variáveis cinemáticas do movimento (STREIT, 2013). 
A origem da análise biomecânica do movimento humano foi discutida antes de Cristo por Aristóteles (370 a.C), Da Vinci (1452-1519), Giovanni Alfonso Borelli (16081679), Muybridge (1831-1904), Marey (1830-1904), conforme pode ser observado na linha do tempo da figura 1. Aristóteles foi o precursor de estudos dos movimentos humano (REY, 2003) e é considerado o Pai da Cinesiologia, sendo o primeiro a descrever o complexo processo da deambulação, na qual o movimento de rotação se transforma em movimento de translação (RASCH, 1991).

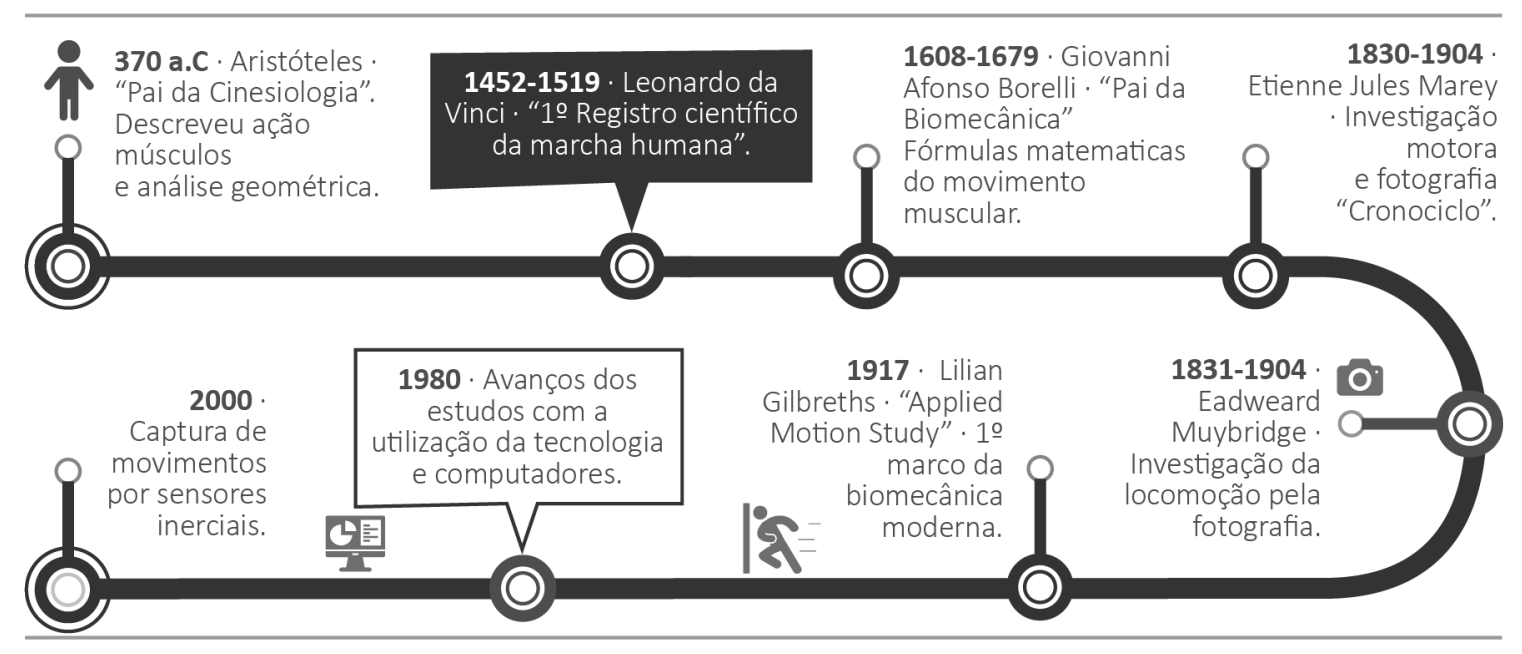

Figura 1: Origem e evolução da Captura de Movimentos

Fonte: Elaborado pelos autores com base em Rasch (1991) e Settineri, Rodrigues (1976).

De acordo com Pinheiro (2008), Leonardo da Vinci (1452-1519) realizou o primeiro estudo sobre anatomia humana contemplando o registro científico da marcha humana no contexto mecânico. Mais tarde, Giovanni Alfonso Borelli (1608-1679), determinou o centro de gravidade do corpo humano, sendo considerado o pai da Biomecânica (MAGARREIRO, 2015). Eadweard Muybridge (1831- 1904) inventou o zoopraxiscópio (dispositivo capaz de reproduzir imagens em movimento), permitindo estudos fotográficos sobre padrões de movimento por meio de uma rede linear de câmeras sequenciais. Inspirado no trabalho de Muybridge, Étienne-Jules Marey (1830-1904) inventou o fuzil cronográfico em 1882, utilizando métodos gráficos e fotográficos capazes de capturar e gravar na mesma imagem 12 frames por segundo (KITAGAWA, 2008).

Não obstante, os estudos sobre o movimento humano em ergonomia encontram-se relacionados ao casal Gilberts (1917), que se dedicou ao estudo de movimentos e fadiga no trabalho (MEDVED, 2001). Já na década de 1970, outros cientistas dedicaram-se às pesquisas da biomecânica e de seus ramos (biomecânica pura, do esporte, reabilitação, eletromiografia, ergonomia), as quais foram produzidas em diferentes laboratórios e publicadas em congressos da área (SETTINERI; RODRIGUES, 1976).

No período de 1980 a 2000, os estudos avançaram com a utilização da tecnologia e dos computadores, possibilitando a redução do tempo de processamentos, armazenamento e representação da informação, aumento da qualidade dos movimentos capturados e uso de sistemas integrados, que permitiram o registro do movimento por meio de dispositivos que possibilitam mensurar as variáveis cinemáticas do movimento (STREIT, 2013). Como exemplo destes dispositivos, tem-se o sistema de captura de movimento 3D Xsens MVN Biomech. 


\subsubsection{Sistema de captura de movimentos - Xsens MVN Biomech}

O Xsens é um sistema baseado em unidades de medida inercial (IMU's), ou seja, modelos biomecânicos e algoritmos de fusão de sensores que combinam sinais tridimensionais provenientes de giroscópio e acelerômetros para rastrear o posicionamento do objeto capturado, determinando sua velocidade, aceleração e posição (XSENS, 2012).

Os giroscópios são utilizados para medir a orientação dos segmentos corporais no espaço 3D, bem como efetuar medições de velocidade de rotação de um objeto em torno de um eixo central. Ou seja, consegue mensurar a velocidade angular servindo como referência de direção, o que permite definir o ângulo e orientação dos objetos (CARVALHO, 2011). Já os acelerômetros são dispositivos eletromecânicos capazes de medir a aceleração e a velocidade própria de objeto, calculando assim as forças exercidas sobre ele (YANG E HSU, 2010). Segundo Lin (2013), os sistemas baseados em acelerômetros são capazes de adquirir e estimar valores cinemáticos (velocidade e o deslocamento) quando ligados aos segmentos do corpo do sujeito.

Neste sentido, o sistema Xsens capta movimentos por meio de 17 módulos de sensores inerciais e magnéticos com frequência de $120 \mathrm{~Hz}$ (gerando 120 frames por segundo), possibilitando a avaliação contínua do movimento dinâmico. Os dados recolhidos são transmitidos por conexão wireless para um computador com software, permitindo a observação, gravação e análise dos movimentos a partir de gráficos dos ângulos das articulações, da velocidade e duração dos movimentos (SPECK et al., 2016; ROETENBER; LUINGE; SLYCKE, 2013). A este respeito, a figura 2, apresenta o posicionamento dos 17 sensores inerciais ao corpo, suas conexões a interface do software utilizado para a captura de dados.

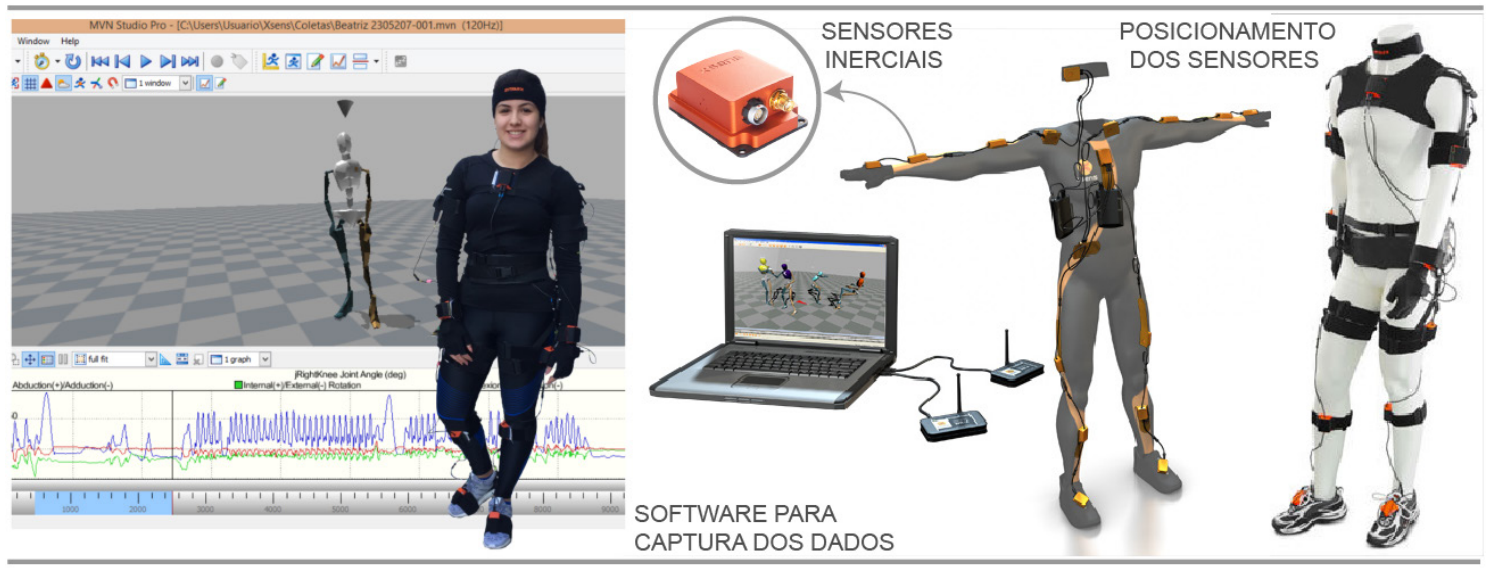

Figura 2: Posicionamento dos sensores inerciais e interface do software Xsens Fonte: Elaborado pelos autores com base em Xsens (2012).

O sistema Xsens permite a captura de qualquer movimento do corpo humano (andar, correr, saltar, rastejar ou fazer reviravoltas) de forma eficaz e em tempo real, dispensando o uso de câmeras externas, emissores ou marcadores. Deste modo, não se limita ao contexto laboratorial, possibilitando estudos e avaliações da execução de atividades em seus contextos reais (XSENS, 2012). O sistema pode ser utilizado ao ar livre e/ou em interiores (habitação, laboratórios), uma vez que não apresenta 
restrições de iluminação nem constrangimentos associados às oclusões ou falta de marcadores (SPECK et al., 2016; ROETENBER; LUINGE; SLYCKE, 2013).

\subsection{Termografia infravermelha}

A termografia infravermelha é uma técnica de detecção da radiação infravermelha que possibilita a medição e o registro das qualidades térmicas de superfícies de objetos e sujeitos, gerando uma imagem térmica, chamada de termograma, que exprime as variações de temperatura por meio de um espectro de cores, que seriam imperceptíveis a olho nu (CERDEIRA et al, 2011; MOBLEY, 2002).

A origem da termografia infravermelha está relacionada às observações feitas pelo médico, filósofo e pesquisador Grego Hipócrates, que identificou variações de temperatura corporais por meio da alteração das características da argila, utilizada como instrumento de avaliação (BRIOSCHI; MACEDO; MACEDO, 2003). Mais tarde, Willians e John Herschel realizaram as primeiras imagens termográficas utilizando o sistema infravermelho por meio da técnica evaporográfica, a qual consiste na evaporação do álcool obtido de uma superfície pintada com carbono (HOLST, 2000). A figura 3 demonstra os principais eventos do desenvolvimento deste método.

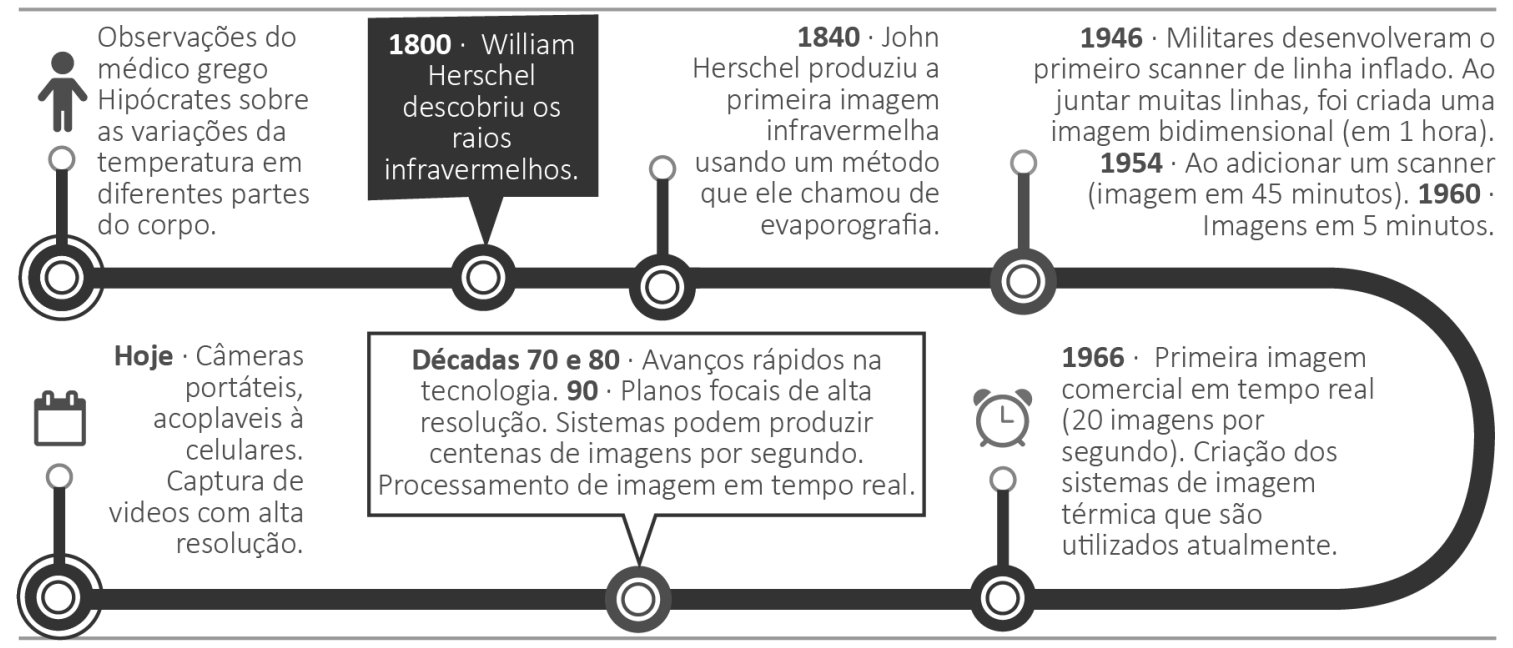

Figura 3: Origem e evolução da Termografia Infravermelha

Fonte: Elaborado pelos autores com base em Brioschi, Macedo e Macedo (2003), Holst (2000) e FLIR (2016b).

No entanto, foram nas décadas de 1970, 1980 e 1990 que os avanços tecnológicos estimularam novos progressos, sendo o maior deles a implementação dos sistemas de alta resolução, capazes de produzir centenas de imagens por segundo e processar a imagem em tempo real (HOLST, 2000). Atualmente, este método possui aporte de diversos modelos de termovisores como os profissionais, os compactos e os adaptados para smartphones, diversidade que possibilita maior acessibilidade e visibilidade a técnica termográfica.

\subsubsection{Sistema da termografia infravermelha}

Todos os objetos emitem constantemente energia térmica para o ambiente, em forma de energia infravermelha, a qual não é visível a olho nu devido ao seu 
comprimento de onda (SILVA, 2017). Nesse sentido, um determinado corpo sob as mesmas condições emitirá a radiação de vários comprimentos de onda, formando um espectro eletromagnético (LANGLEY, 1985).

Estas ondas, também chamadas de bandas, são distinguidas pelos métodos de detecção de radiação, possibilitando as imagens térmicas. O termovisor é a ferramenta utilizada para realizar a captura, sendo capaz de detectar o infravermelho em uma escala do espectro eletromagnético de aproximadamente 2.000-13,000 nanômetros ou 2-13 $\mu \mathrm{m}$. (FLIR, 2016b). Esta tecnologia funciona por meio da captura da energia infravermelha emitida pelos objetos e sujeitos, a qual não é visível a olho nú (SILVA, 2017), conforme demonstrado na figura 4.

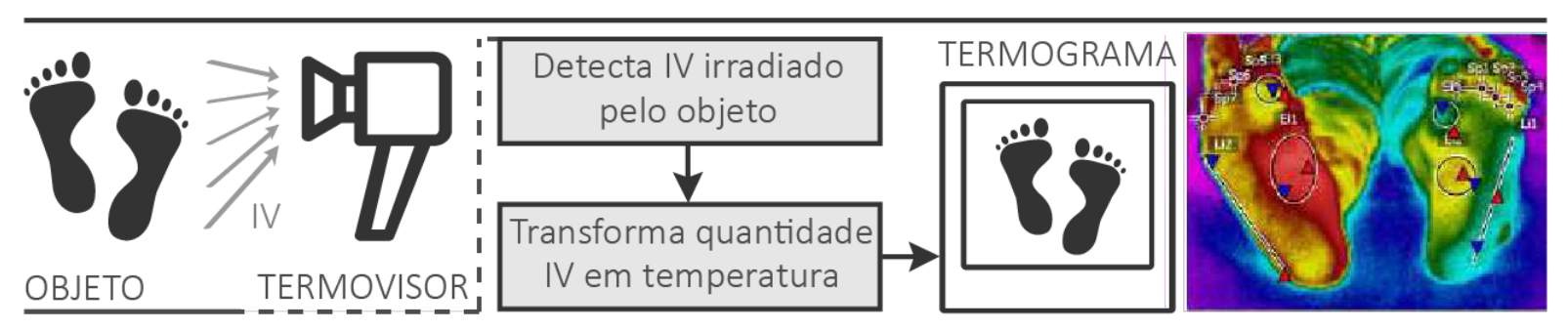

Figura 4: Processo da captura termográfica Fonte: Adaptado de SILVA (2017).

Assim, esse mapeamento possibilita a visualização de áreas e variações de emissão de calor da superfície da matéria em estudo.

\section{PROCEDIMENTOS METODOLÓGICOS}

Esta pesquisa caracteriza-se como aplicada, que segundo Lakatos e Marconi (2011) tem por objetivo gerar conhecimentos para aplicação prática, buscando a solução de problemas específicos e envolvendo verdades e interesse local. Dessa forma, o artigo visa apresentar informações sobre o uso da instrumentação tecnológica de captura de movimentos (Xsens) e de termografia infravermelha na coleta de dados em estudos de TA e também em pesquisas cientificas gerais.

Quanto à abordagem, utilizou-se a pesquisa qualitativa que, segundo Creswell (2010, p. 26), "é um meio para explorar e entender o significado que os indivíduos ou grupos atribuem a um problema social ou humano". Deste modo, a pesquisa buscou compreender a captura de movimentos e a termografia infravermelha, destacando sua utilização nas coletas de dados para o desenvolvimento de projetos de TA.

De acordo com seus objetivos, classifica-se como descritiva pois se propõe a apresentar casos que utilizaram a instrumentação tecnológica - Xsens e termografia infravermelha - na obtenção de dados, apresentando projetos desenvolvidos pelo NGD/LDU. Segundo Sampieri, Collado e Lucio (2013), os estudos descritivos buscam especificar as propriedades, as características e o perfil de pessoas, grupos, comunidades, processos, objetos ou qualquer outro fenômeno que se submeta a uma análise. São úteis para mostrar com precisão os ângulos ou dimensões de um fenômeno, acontecimento, comunidade, contexto ou situação. 


\subsection{Etapas de pesquisa}

A pesquisa segmenta-se em duas etapas: etapa 1 - Levantar; etapa 2 - Analisar e identificar. A primeira etapa (Levantar) contempla a realização da pesquisa bibliográfica, elaborada a partir de materiais já publicados, sobre o tema proposto - captura de movimentos e termografia infravermelha aplicadas à projeto de TA - e da pesquisa documental dos projetos de TA desenvolvidos pelo NGD/LDU no período de 2015 a 2017. Na segunda etapa (Analisar e Identificar) foi realizada a análise dos documentos levantados na etapa anterior com o intuito de identificar os protocolos, vantagens e desvantagens dos mesmos.

\subsection{Materiais e Métodos}

Na etapa 1 (Levantar), foi realizado o levantamento bibliográfico, nos meses de outubro e novembro de 2017. Este levantamento ocorreu de forma assistemática com base em teses e dissertações, bem como artigos de periódicos nacionais e internacionais. A pesquisa documental, ocorreu no mesmo período no acervo de dados do NGD/LDU, referente a projetos desenvolvidos no período de 2015 a 2017.

Posteriormente, na etapa 2 (Analisar e identificar), os documentos foram selecionados tendo como critérios de inclusão: estudos que abordavam a utilização da instrumentação tecnológica - captura de movimentos e termografia infravermelha. Após esta seleção, foi realizado a extração dos dados, por meio de tabelas, identificando os protocolos, vantagens e desvantagens da instrumentação tecnológica. Por fim, foi realizado a organização e apresentação dos resultados.

\section{RESULTADOS}

Neste tópico são apresentados os protocolos, vantagens e desvantagens a respeito da utilização da captura de movimentos (Xsens) e a termografia infravermelha em estudos de casos de TA, desenvolvidos pelo NGD/LDU, apresentados nos artigos de Merino et al. (2017); Speck et al. (2016) e García et al. (2016) como também de projetos de pesquisas gerais.

\subsection{Captura de movimento}

No que tange a utilização de protocolos para a coleta de dados com o Xsens, Speck et al. (2016), relataram que para o desenvolvimento do estudo em TA de uma órtese para o joelho, utilizou-se da instrumentação integrada para a coleta de dados eletromiografia, dinamometria, termografia infravermelha e captura de movimentos por sensores inerciais (Xsens). Com o aporte de uma equipe multidisciplinar, realizou-se a análise das atividades de marcha na barra paralela e bicicleta ergométrica. Os autores relatam que a coleta com o Xsens seguiu o protocolo nativo do próprio equipamento - colocação, medição e calibração, com gravação em $120 \mathrm{~Hz}$. Neste caso, salientam a necessidade da realização das medidas e calibrações do paciente 
na posição sentada e deitada, contando com o auxílio do fisioterapeuta devido as limitações do sujeito, um paciente com demência mista e limitações de movimentos nos membros inferiores decorrentes das doenças de Parkinson e Alzheimer.

Não obstante, Merino et al. (2017) relatam outro estudo em TA com o desenvolvimento de uma órtese para estabilização da articulação do tornozelo, visando auxiliar na correção das disfunções de movimento do membro afetado. Para a coleta de dados, também se utilizou da instrumentação tecnológica integrada (captura de movimentos, termografia infravermelha, dinamometria, eletromiografia). Neste estudo, a captura de movimentos (Xsens) foi utilizada com a finalidade de analisar o processo de marcha do paciente, conforme figura 5.

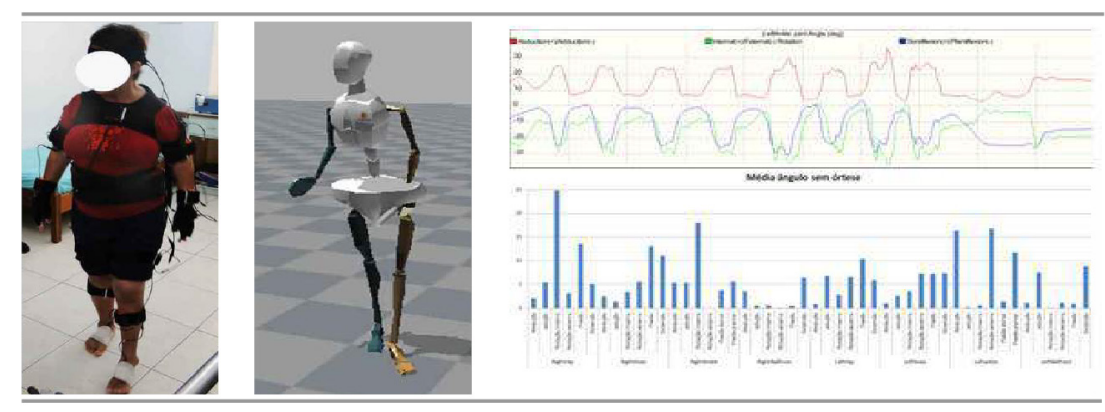

Figura 5: Captura e análise de dados da marcha do sujeito Fonte: Adaptado de García et al. (2016).

O protocolo utilizado foi o protocolo nativo do próprio equipamento, como no estudo de Speck et al. (2016). Os autores afirmam que o procedimento possibilitou compreender e obter informações quantitativas dos ângulos de movimento, velocidade dos segmentos corporais, frequência de movimentos e deslocamento do centro de massa, o que possibilitou gerar alternativas de modelos volumétricos, para serem confeccionados em PVC e testados com o usuário (GARCÍA et al., 2016; MERINO et al., 2017).

Os estudos de Saber-Sheikh et al. (2010); Dinu et al. (2012); Zhang et al. (2013) demonstraram que o Xsens é um método propício para avaliar os parâmetros da cinemática 3D da marcha, devido a sua precisão e confiabilidade durante o registro das informações. Os estudos apresentados apontaram vantagens referentes ao sistema, destacando sua capacidade de estimar a orientação de cada segmento do corpo e das alterações da sua posição, com 6 graus de liberdade, por integração contínua dos sinais do acelerômetro e do giroscópio que atualizam o modelo biomecânico com taxa máxima de $120 \mathrm{~Hz}$, possibilitando uma avaliação contínua do movimento.

A este respeito, Zhang et al (2013), utilizando o protocolo nativo, buscou validar o sistema Xsens em um estudo comparativo com o sistema ocular de captura de movimento (sistemas de câmaras laboratoriais) baseado em câmera de medição da cinemática angular das articulações. Diante disso, o autor destaca a capacidade do Xsens em recolher dados cinemáticos 3D no plano sagital para as articulações do quadril, joelho e tornozelo, recolhendo com precisão, dados de flexão e extensão das três articulações, durante a marcha em superfície plana, e em atividades de subir e descer degraus.

Tonin et al. (2014) aborda os potenciais benefícios do uso integrado de ferramentas computacionais de simulação humana, apresentando uma análise comparativa de siste- 
mas de captura de movimentos (inerciais, mecânicos, ópticos, magnéticos e markerless). Neste estudo foi analisada a liberdade de movimentos durante a captura, a flexibilidade no ambiente de uso e a integração com o software de modelagem e simulação humana. Os resultados mostraram que todos os sistemas (inerciais, mecânicos, ópticos, magnéticos e markerless) analisados apresentam limitações quanto aos critérios estabelecidos, sendo que a tecnologia inercial foi a que melhor atendeu aos critérios devido a sua facilidade de uso, portabilidade e avaliação contínua do movimento.

O estudo de Salvalaio (2012) teve como objetivo contribuir com a captura de movimento angular, evidenciando uma base tecnológica ao design de produtos assistivos, especificamente do controle versátil de cadeiras de rodas motorizadas a pessoas com deficiência motora e severa. Para tanto, foram realizadas capturas de movimento por sistemas ópticos e não ópticos como rastreamento eletromagnético e inercial. De acordo com o autor, o Xsens apresenta limitações como a quantidade de fios presos aos sensores e a dificuldade no ajuste dos sensores sobre o corpo, justificando que a precisão da captura depende do exato posicionamento sobre o vértice das articulações.

Apesar de ser um sistema de análise cinemática tridimensional, o Xsens apresenta limitações em relação a escolha de dados nos planos do movimento (coronal e transversal), razão pela qual se justifica a utilização no plano sagital, onde revela níveis elevados de validade (NOVAK et al., 2014; ZHANG et al., 2013; ROETENBER; LUINGE; SLYCKE, 2013). Consoante a isso, Novak et al. (2014) e Wentink et al. (2014) salientam que o sistema Xsens é satisfatório, mesmo com a limitação no que se refere à validação do sistema nos planos de movimento (coronal e transversal), quando comparado com a maioria dos algoritmos de análise de marcha.

Carvalho (2011) aborda que os sensores inerciais estão sujeitos a desvios (denominados drift) que diminuem a qualidade da captura realizada, causando erros nos dados e consequentemente diminuindo a acurácia dos movimentos capturados com relação aos reais. $O$ autor justifica que esse fato ocorre devido a imprecisões do fator de escala, desalinhamento e ruído, que evoluem com o tempo de captura e são intrínsecos ao processo. No entanto, recomenda-se a utilização dos magnetômetros, que permitem a redução dos erros e do ciclo de captura das gravações, garantindo maior confiabilidade nos dados obtidos (CARVALHO, 2011; SABER-SHEIKH et al., 2010; DINU et al., 2012; ZHANG et al., 2013).

\subsection{Termografia infravermelha}

No que tange o protocolo de levantamento de dados por meio da termografia infravermelha, diversas variáveis devem ser consideradas e controladas para garantir a confiabilidade dos dados obtidos. Neste sentido, Fernández-Cuevas et al. (2015) destaca três fatores que podem influenciar no registro termográfico com sujeitos: ambientais, técnicos e individuais.

Quanto aos fatores ambientais, Fernández-Cuevas et al. (2015) destaca a influência de aspectos como temperatura, umidade, tamanho da sala, pressão atmosférica, radiação, entre outros. A influência destes fatores nas medições pode comprometer os experimentos (SILVA; TARALLI; MELZ, 2015). 
No projeto da órtese para estabilização do joelho, mencionado anteriormente, analisou-se por meio da termografia infravermelha a região da lombar associada a atividade da marcha na barra paralela e da bicicleta ergométrica (MERINO et al., 2017; SPECK et al., 2016). Já o projeto da órtese para estabilização do tornozelo utilizou as medições termográficas para coletar dados dos pés e pernas, antes e após a atividade da marcha (GARCÍA et al., 2016). As imagens dos dois projetos desenvolvidos foram captadas em ambiente controlado com temperatura média de $22^{\circ} \mathrm{C}$ e a câmera foi posicionada a 1 metro do sujeito, posicionado para registro da região investigada, antes e após as atividades (GARCÍA et al., 2016; MERINO et al, 2017; SPECK et al., 2016).

No que se refere aos fatores técnicos, Fernández-Cuevas et al. (2015) apontam os aspectos relacionados ao uso e capacidades do equipamento. Nesse sentido, destaca-se o uso dos softwares dos fabricantes dos termovisores, utilizados pelos pesquisadores para a análise dos dados. Em ambos os projetos das órteses, utilizou-se o software para padronizar e analisar a escala cromática (Rainbow HC), o grau de emissividade $(0,98)$, a temperatura de reflexão $\left(20^{\circ} \mathrm{C}\right)$ e com intervalo de temperatura, que foi de $15^{\circ} \mathrm{C}$ a $40^{\circ} \mathrm{C}$ (para órtese do joelho) e $25^{\circ} \mathrm{C}$ a $40^{\circ} \mathrm{C}$ (para órtese do tornozelo) (GARCÍA et al., 2016; MERINO et al, 2017; SPECK et al., 2016).

Quanto aos fatores individuais citados por Fernández-Cuevas et al. (2015) são segmentados em aspectos intrínsecos e extrínsecos dos sujeitos. Os intrínsecos são relativos ao sexo, idade, genética, entre outros, enquanto os extrínsecos estão relacionados às atividades praticadas e os tratamentos. Nos estudos citados foram consideradas as características dos sujeitos, que apresentavam mobilidade reduzida, um apresentando demência e limitações de movimentos nos membros inferiores (SPECK et. al, 2016) e outro com força muscular reduzida do membro inferior esquerdo (GARCÍA et al., 2016)

As análises dos resultados dos estudos das órteses visaram comparar características do sujeito antes e após as atividades, verificando a diferença térmica entre os pontos e presença de assimetria térmica segundo critérios de Brioschi et al. (2002). O controle destes fatores foi essencial para comparar os resultados do mesmo usuário antes e após a atividade (GARCÍA et al., 2016; MERINO et al, 2017; SPECK et al., 2016). García et al. (2016), por meio da termografia infravermelha, obteve informações que possibilitaram a identificação das áreas do pé do sujeito, onde foram necessários reforços acolchoados para evitar atritos e dores durante a atividade da marcha.

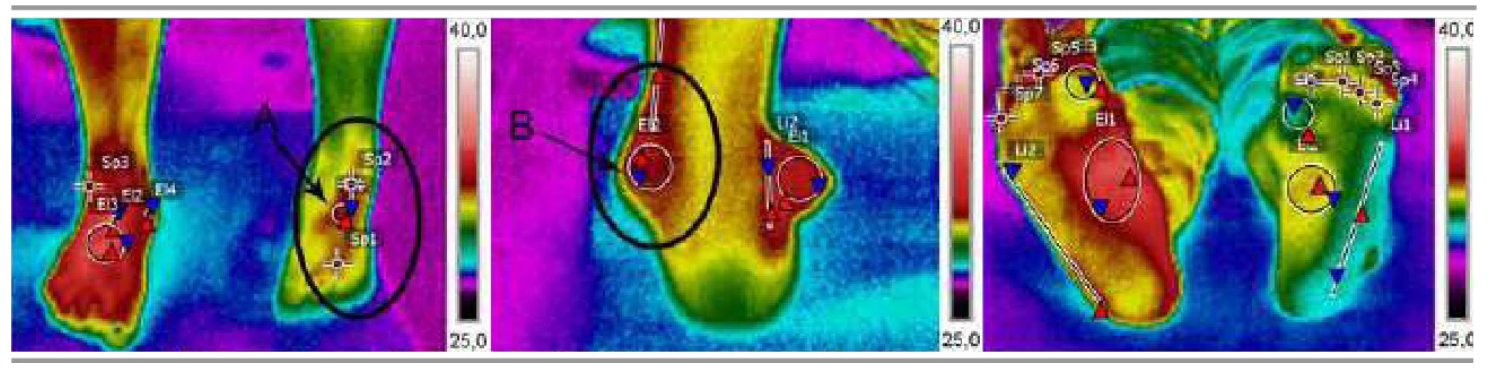

Figura 6: Imagens termográficas dos pés e tornozelos do sujeito Fonte: Adaptado de García et al. (2016). 
Na pesquisa de Medeiros et. al (2015), que comparou variáveis subjetivas e objetivas do desconforto de cadeirantes na subida de rampas, destacou-se a necessidade do entendimento da fisiologia humana para a obtenção de dados nos momentos corretos. Nesse sentido, destaca-se a necessidade de um período de estabilização da temperatura corporal após a atividade, para então realizar o registro termográfico.

No que se referem suas vantagens, a termografia infravermelha permite uma exploração aprofundada e menos subjetiva dos sujeitos e objetos de estudo, possibilitando a identificação de um quadro visual para comparação de temperaturas, bem como a capacidade da identificação de deteriorações nos produtos por meio de componentes aquecidos (SILVA; TARALLI; MELZ, 2015). Giorleo e Meola (2002) corroboram com os autores e destacam ainda o caráter bidimensional da técnica, que é utilizada na medição do campo de temperatura da superfície de todos os tipos de materiais.

Não obstante, caracteriza-se como um método de medição não destrutivo, passivo e sem contato direto para o mapeamento térmico de um corpo por meio da radiação infravermelha emitida pelas superfícies (BRIOSCHI; MACEDO; MACEDO, 2003; HOLST, 2000; SILVA; TARALLI; MELZ, 2015). De acordo com Silva, Taralli e Melz (2015), a técnica permite a detecção de defeitos em materiais e possui compatibilidade com diversos softwares para a interpretação das imagens. Assim, por meio da instrumentação, torna-se possível observar sujeitos e objetos de maneira confiável em situações diversas, inclusive quando a falta de comunicação dificulta o acesso à informações (MERINO et al., 2017; SPECK et al., 2016).

Silva (2017) apresenta vantagens do uso da termografia infravermelha nos setores industriais, destacando a possibilidade de detecção rápida de problemas, aumento dos rendimentos e da segurança dos sistemas, a redução dos custos de manutenção, custos operacionais e associados à imprevistos. Para Silva, Taralli e Melz (2015) suas aplicações podem auxiliar no diagnóstico do mau uso de materiais e peças, falhas de projeto, bem como de percepções dos usuários, proporcionando o redesign de produtos.

Quanto às desvantagens da termografia infravermelha, Silva, Taralli e Melz (2015) destacam a limitação da detecção de apenas temperaturas superficiais, as interferências das condições do ambiente nas medições, bem como a dificuldade na interpretação das imagens de determinados materiais devido sua emissividade. Além disso, os investimentos em equipamentos, os valores elevados das câmeras termográficas de alta qualidade e a precisão limitada de algumas são citados como desvantagens (SILVA, 2017; SILVA; TARALLI; MELZ, 2015). Silva (2017) também destaca a necessidade do envolvimento de profissionais capacitados nos projetos, com conhecimentos técnicos específicos sobre o uso desta tecnologia.

\subsection{Síntese dos resultados}

Em suma, diante dos resultados apresentados, elaborou-se a figura 7, que apresenta uma síntese visual sobre as principais características dos protocolos, bem como as vantagens e desvantagens de cada equipamento. 


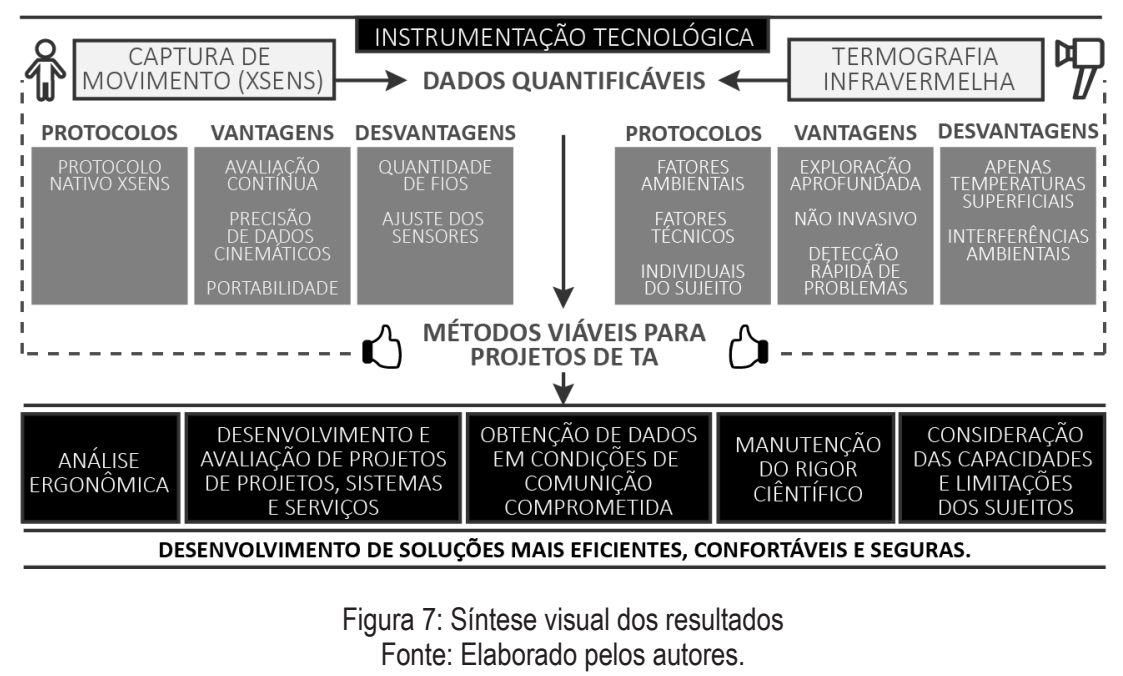

Diante do exposto, entende-se que a instrumentação tecnológica (captura de movimentos e a termografia infravermelha) possibilita informações e definições quantificáveis, por meio da utilização de protocolos rigorosos. Assim, tornam-se métodos viáveis para projetos de TA, possibilitando análises ergonômicas, desenvolvimento e avaliações finais de projetos de produtos, sistemas e serviços, mesmo em condições onde a comunicação com o sujeito encontra-se comprometida. Desta forma, atende à necessidade de rigor científico dos pesquisadores e respeita as capacidade e limitações dos usuários, auxiliando no desenvolvimento de soluções mais eficientes, confortáveis e seguras.

\section{CONCLUSÕES}

Diante dos resultados obtidos foi possível compreender como a instrumentação tecnológica está sendo utilizada no levantamento de dados para o desenvolvimento de projetos de TA, auxiliando na obtenção de medições objetivas (quantitativas), gerando dados sobre os sujeitos e objetos analisados, bem como sobre problemas relacionados ao conforto, segurança e usabilidade. Em projetos de TA, a instrumentação se mostra adequada para as coletas de dados envolvendo pessoas com limitações físicas e psíquicas, mantendo o rigor científico sem desconsiderar as necessidades do usuário e do projeto (MERINO et al., 2017; SPECK et al., 2016).

No entanto, por meio realização da pesquisa bibliográfica e documental dos projetos NGD/LDU, foi possível identificar protocolos, vantagens e desvantagens a respeito da utilização da captura de movimentos (Xsens) e a termografia infravermelha. Nesse sentido, os procedimentos adotados se mostraram satisfatórios para a obtenção dos resultados.

Diante dos estudos apresentados, percebe-se que os métodos criteriosos de experimentos científicos são considerados nas comunidades científicas, a fim de garantir a reprodutibilidade das pesquisas por diferentes grupos de pesquisadores. Para tanto, torna-se necessária a adequação e o controle de um conjunto de variáveis no desenvolvimento dos projetos. Neste sentido Merletti e Parker (2004) apontam que esforços de padronização são necessários para a geração de resultados que pos- 
sam ser comparados e/ou repetidos, a fim de se a criar uma base de conhecimentos comum sobre um determinado campo da ciência.

A tecnologia da captura de movimentos permite aos pesquisadores superar as limitações da simulação humana tradicional, permitindo a avaliação de produtos e atividades, bem como a análise de interações entre homem e objeto. Ainda, reduz o tempo para simulações atribuindo maior realidade aos movimentos simulados. Consoante a isso, a termografia infravermelha permite a compreensão do estado muscular do sujeito, bem como auxilia nas etapas de desenvolvimento e avaliação de produtos, auxiliando na obtenção de recursos adicionais aos procedimentos de mapeamento térmico, permitindo uma exploração aprofundada e menos subjetiva. Convém destacar, que o uso desta técnica não é invasiva e não possui contraindicações, auxiliando no uso de pacientes com limitações físicas e psíquicas.

Esta pesquisa demonstrou que a aplicação da instrumentação tecnológica requer o suporte do conhecimento de equipes multidisciplinares, principalmente em projetos envolvendo sujeitos com limitações, onde os profissionais da saúde contribuem no processo de obtenção e análise dos dados. Os instrumentos tecnológicos e procedimentos de coletas de dados aproximam o designer das necessidades reais do usuário, suas limitações e possibilidades, fazendo com que os projetos sejam mais efetivos e promovam melhorias na qualidade de vida de pessoas com deficiência ou mobilidade reduzida.

Por meio desta pesquisa não foram identificados protocolos específicos para projetos de TA, no entanto, sugere-se como futuros estudos, o desenvolvimento de protocolos adequados e focados neste tipo de projeto, visando o aprimoramento dos roteiros de coletas com a instrumentação integrada.

\section{AGRADECIMENTOS}

Agradecemos ao Programa de Pós-graduação em Design da UFSC (PPGD/ UFSC), ao Núcleo de Gestão de Design e Laboratório de Design e Usabilidade (NGD-LDU/UFSC), à Pró-Reitoria de Extensão (PROEX/UFSC), à Fundação de Amparo à Pesquisa e Extensão Universitária (FAPEU), à Rede de Pesquisa e Desenvolvimento em Tecnologia Assistiva (RPDTA), ao Instituto Psiquiátrico de Santa Catarina (IPQ/SC), à Coordenação de Aperfeiçoamento de Pessoal de Nível Superior (CAPES), ao Conselho Nacional de Desenvolvimento Científico e Tecnológico (CNPq), à Secretaria de Educação Superior (SESu), ao Ministério da Educação (MEC) e aos demais envolvidos.

\section{REFERÊNCIAS}

BRASIL. Cartilha do Censo 2010: Pessoas com deficiência. Secretária de Direitos Humanos da Presidência da República (SDH-PR); Secretaria Nacional dos Direitos da Pessoa com Deficiência (SNPD), et al. Brasilia: SDH-PR/SNPD, 2012.

BRASIL. Lei 13.146 de 06 de julho de 2015. Institui a Lei Brasileira de Inclusão da Pessoa com Deficiência (Estatuto da Pessoa com Deficiência). Diário Oficial da União, 2015. 
BRIOSCHI, Marcos L.; MACEDO, José F; MACEDO, Rodrigo de A. C. Termometria cutânea: novos conceitos. Jornal Vascular Brasileiro, v. 2, p. 151-60, 2003.

CARVALHO, Adriano G. Influência da modelagem dos componentes de bias instabilidade dos sensores inerciais no desempenho do navegador integrado SNI/GPS. Instituto Militar de Engenharia, Rio de Janeiro. 2011.

CERDEIRA, Fernando et al. Applicability of infrared thermography to the study of the behaviour of stone panels as building envelopes. Energy and Buildings, v. 43, n. 8, p. 1845-1851, 2011.

CRESWELL, John W. Projeto de pesquisa: Métodos qualitativo, quantitativo e misto. 3 ed. Porto Alegre: Artmed, 2010.

DINU, Daniel et al. Accuracy of Postural Human-motion Tracking Using Miniature Inertial Sensors. Procedia Engineering, v. 147, p. 655-658, 2016.

FERNÁNDEZ-CUEVAS, Ismael et al. Classification of factors influencing the use of infrared thermography in humans: A review. Infrared Physics \& Technology, v. 71, p. 28-55, 2015.

FLIR. 3 principais aplicações da análise termográfica. (2016a). Disponível em: <http:// www.flir.com.br/home/news/details/?ID=80767>. Acesso em: 25 set. 2017.

FLIR. Manual do utilizador. Série FLIR Exx. (2016b). Disponível em: <http://support.flir. com>. Acesso em: 25 set. 2017.

GARCÍA, David N. et al. Projeto de órtese: Integração de duas sistemáticas de desenvolvimento de projetos aplicadas a TA. Anais de congresso. 1을 Congresso Brasileiro de Pesquisa e Desenvolvimento em Tecnologia Assistiva (CBTA), Curitiba/PR, Brasil, 2016.

GOMIDE, João Victor Boechat et al. Captura de movimento e Animação de Personagens em Jogos. In: Brazilian Symposium on Games and Digital Entertainment, 8., 2009, Rio de Janeiro. Simpósio... Rio de Janeiro: PUC, 2009.

HOLST, Gerald C. Common sense approach to thermal imaging. Washington, DC, USA: SPIE Optical Engineering Press, 2000.

LAKATOS, Eva Maria; MARCONI, Marina de Andrade. Fundamentos de metodologia científica. 6.ed., São Paulo, Atlas, 2011

LÄMKULL, Dan; HANSON, Lars; ÖRTENGREN, Roland. A comparative study of digital human modelling simulation results and their outcomes in reality: A case study within 
manual assembly of automobiles. International Journal of Industrial Ergonomics, v. 39, n. 2, p. 428-441, 2009.

LANGLEY, Billy C. Comfort heating. 3rd ed. Reston: Reston Publishing, 1985.

LIN, Jonathan Feng-Shun. Automated Rehabilitation Exercise Motion Tracking. 2013. 112 f. Dissertação (Mestrado) - Curso de Pós-graduação em Engenharia Elétrica e Informática, University of Waterloo, Waterloo, 2013.Disponível em: < https://uwspace.uwaterloo.ca/bitstream/handle/10012/7191/Lin_Jonathan.pdf?sequence=1 >. Acesso em: 05 jan. 2018.

MAGARREIRO, João. Protocolo de recolha e processamento de dados 3D cinemáticos e cinéticos da marcha em regime laboratorial e ambulatório. 2015. 105 f. Dissertação (Mestrado) - Curso de Pós-graduação em Engenharia Biomédica- Desporto e Reabilitação, Instituto Politécnico de Setúbal, Setúbal, 2015. Disponível em: < https:// comum.rcaap.pt/bitstream/10400.26/8236/1/Dissertacao_MEBDR_ESTSetubal_ Joao_Magarreiro_120289014.pdf >. Acesso em: 05 jan .2018.

MEDEIROS, Ivan L. et al. Avaliação Ergonômica do Desconforto Muscular causado pelo uso de Cadeira de Rodas. Human Factors in Design, v. 4, n. 8, p. 095-111, 2015. Disponível em: <http://www.revistas.udesc.br/index.php/hfd/article/view/6642/4755>. Acesso em: 18 dez. 2017.

MERINO, Eugenio et al. Implementation of Integrated Instrumentation in Assistive Technology. Advances In Ergonomics In Design, [s.l.], p.549-560, 24 jun. 2017. Springer International Publishing. http://dx.doi.org/10.1007/978-3-319-60582-1_55.

MERLETTI Roberto, PARKER Philip. A. Eletromyography: Physiology, Engineering and Noninvasive applications. New York. Wiley-IEEE Press, 2004.

MOBLEY, R. Keith. An introduction to predictive maintenance. 1. ed. Amsterdam: Butterworth-Heinemann, 2002.

NGD/LDU (Núcleo de Gestão de Design e Laboratório de Design e Usabilidade). Sobre o NGD/LDU. Universidade Federal de Santa Catarina, Florianópolis, 2017. Disponível em: < https://ngdldu-ufsc.squarespace.com/ngdldu// >. Acesso em: 07 jan. 2018.

NOVAK, Domen et al. Toward real-time automated detection of turns during gait using wearable inertial measurement units. Sensors, v. 14, n. 10, p. 18800-18822, 2014.

PINHEIRO, Alan Petrônio. Desenvolvimento de um sistema de captura e análise de movimentos baseado em técnicas de visão computacional. 2008. 125 f. Dissertação (Mestrado) - Curso de Pós-graduação em Engenharia Elétrica, Universidade Federal de Uberlândia, Uberlândia, 2008. Disponível em: < http://repositorio.ufu.br/bitstream/123456789/14376/1/Alan.pdf>. Acesso em: 04 jan. 2018. 
RASCH, Philip J. Cinesiologia e anatomia aplicada. 7. ed. Rio de Janeiro: Guanabara Koogan, 1991.

REY, Puente Fernando.Télos como arché e o fundamento temporal da ação em Aristóteles. Revista Philosophica 26. Instituto de Filosofía Pontificia Universidad Católica de Valparaíso: 2003.

ROETENBERG, Daniel; LUINGE, Henk; SLYCKE, Per. Xsens MVN: full 6DOF human motion tracking using miniature inertial sensors. Xsens Motion Technologies BV, Tech. Rep, 2013.

SABER-SHEIKH, Kambiz et al. Feasibility of using inertial sensors to assess human movement. Manual Therapy, v. 15, n. 1, p. 122-125, 2010.

SALVALAIO, Cláudio Luiz. Contribuição ao estudo da captura do movimento aplicado ao design em tecnologia assistiva. 2012. 122 f. Dissertação (Mestrado) - Curso de Pós-design, Engenharia de Materiais, Universidade Federal do Rio Grande do Sul, Porto Alegre, 2012. Disponível em: <http://www.lume.ufrgs.br/handle/10183/62049>. Acesso em: 06 jan. 2018.

SAMPIERI, Roberto Hernández; COLLADO, Carlos Fernández; LUCIO, María del Pilar Baptista. Metodologia de pesquisa. 5.ed. Porto Alegre: Penso, 2013

SETTINERI, Luiz Irineu Cibilis; RODRIGUES, Raul Barnech. Fundamentos de cinesiologia. Porto Alegre: Movimento/Universidade Federal do Rio Grande do Sul, 1976.

SILVA, Júlio Cézar R. P. da; TARALLI, Cibele H.; MELZ, Simone P. M. Termograma: A imagem térmica como instrumento de diagnóstico rápido no design. In: Fourth International Conference on Integration of Design, Engineering and Management for innovation. Anais... Florianópolis, SC, Brasil: 2015. Disponível em: <http://janainaramos.com.br/idemi2015/anais/02/143332.pdf>. Acesso em: 10 jan. 2018.

SILVA, Luiz A. da. Termografia: Princípios básicos e suas aplicações. 1. ed. São José dos Campos: Tecnolass Tecnologia, 2017.

SPECK, Giselle Mari et al. Processo de instrumentação integrada no desenvolvimento de projetos de Tecnologia Assistiva. Anais de congresso. 18ํㅡㄴ Congresso Brasileiro de Ergonomia (ABERGO), Belo Horizonte: 2016.

STREIT, Priscilla. Comparação de parâmetros biomecânicos entre sistemas de captura de movimentos: avaliação do Microsoft Kinect. 2013. 98 f. Dissertação (Mestrado) Curso Pós-graduação em Design, da Universidade do Estado do Rio de Janeiro, Rio de Janeiro, 2013. Disponível em: http://bdtd.ibict.br/vufind/Record/UERJ_c6d8f4f128ed6ba8dc3e8174e0d59c26 >. Acesso em: 06 jan.2018. 
TONIN, Luiz Antônio et al. Avaliação de tecnologias de captura de movimentos em projetos de situações produtivas. Anais de congresso. 17ํㅡㄹ Congresso Brasileiro de Ergonomia (ABERGO), Rio de Janeiro: 2014.

WHO. Relatório mundial sobre a deficiência. SEDPcD. São Paulo, p.334. 2012

WEENK, Dirk et al. Automatic identification of inertial sensor placement on human body segments during walking. Journal of neuroengineering and rehabilitation, v. 10, n. 1, p. 31, 2013.

XSENS. Moven: user manual. Moven Motion Capture System. The Netherlands: Xsens Technologies B.V. 2012.

YANG, Che-Chang; HSU, Yeh-Liang. A review of accelerometry-based wearable motion detectors for physical activity monitoring. Sensors, v. 10, n. 8, p. 7772-7788, 2010.

ZHANG, Jun-Tian et al. Concurrent validation of Xsens MVN measurement of lower limb joint angular kinematics. Physiological measurement, v. 34, n. 8, p. N63, 2013. 